\title{
Tape-Stripping Electrochemical Detection of
}

\author{
Melanoma
}

\section{- Supplementary Material -}

Sorour Darvishi, ${ }^{[a]}$ Horst Pick, ${ }^{[b]}$ Tzu-En Lin, ${ }^{[c]}$ Yingdi Zhu, ${ }^{[a]}$ Xiaoyun Li, ${ }^{[d, e]}$ Ping-Chih Ho, ${ }^{[d, e]}$

Hubert H. Girault, ${ }^{*[a]}$ and Andreas Lesch $*[f]$

${ }^{a}$ Laboratory of Physical and Analytical Electrochemistry, Ècole Polytechnique Fédérale de Lausanne (EPFL) Valais Wallis, Rue de l'Industrie 17, CH-1950 Sion, Switzerland.

b Institute of Chemical Sciences and Engineering, Ècole Polytechnique Fédérale de Lausanne (EPFL), EPFL Station 15, CH-1015 Lausanne, Switzerland.

c Institute of Biomedical Engineering, College of Electrical and Computer Engineering,National Chiao Tung University, Hsinchu 30010, Taiwan

${ }^{d}$ Department of Oncology, University of Lausanne, Ch. des Boveresses 155, CH-1066 Epalinges, Switzerland.

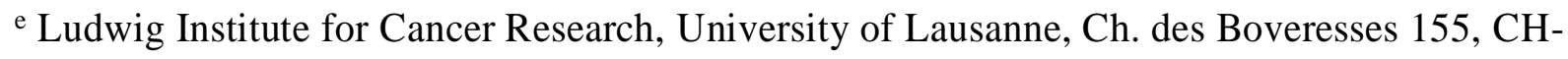
1066 Epalinges, Switzerland.

f Department of Industrial Chemistry "Toso Montanari", University of Bologna, Viale del Risorgimento 4, IT-40136 Bologna, Italy.

\section{${ }^{*}$ CORRESPONDING AUTHOR FOOTNOTE}

EMAIL: hubert.girault@epfl.ch; andreas.lesch@unibo.it 


\section{List of supplementary information:}

SI-1. Sample preparation of cells on adhesive tape.

SI-2. Microscopic images of melanoma cells.

SI-3. Soft microelectrode and schematic representation of SECM line scans on the taped samples.

SI-4. Cyclic voltammogram of a soft microelectrode in FcMeOH solution.

SI-5. Soft-Probe-SECM line scans over cells on adhesive tape with statistical analysis.

SI-6. Colorimetric TYR visualization with TMB of human melanoma cell lines.

SI-7. Colorimetric TYR visualization with TMB substrate for viable melanoma cell detection with intact cell membranes. 


\section{SI-1. Sample preparation of cells on adhesive tape.}

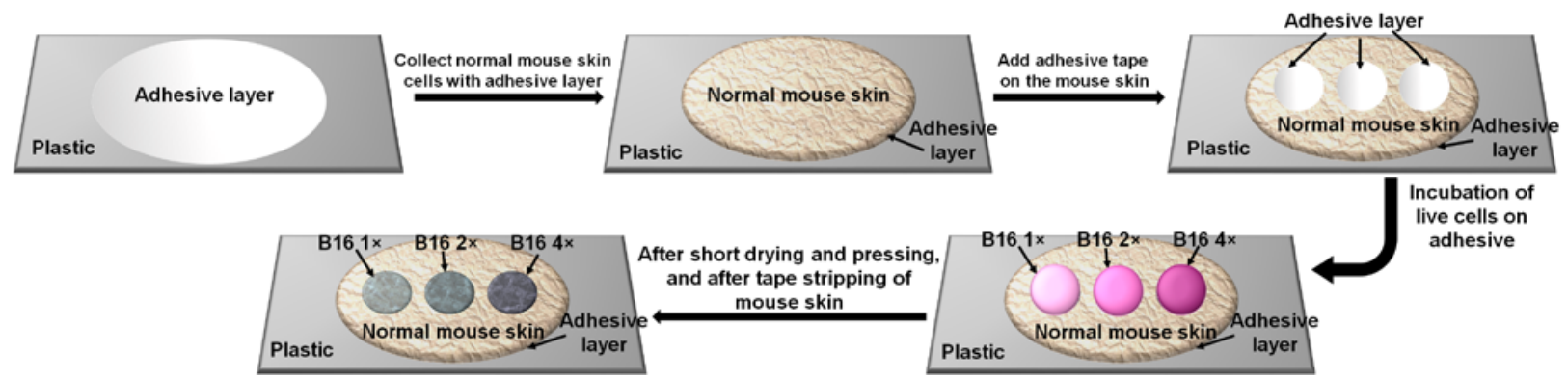

Figure S-1. Schematic of exemplary sample preparation of a tape sample with B16 melanoma cells, previously grown in Petri dishes and dropped on the adhesive layers; normal mouse skin was collected from the melanoma-free back of mice.

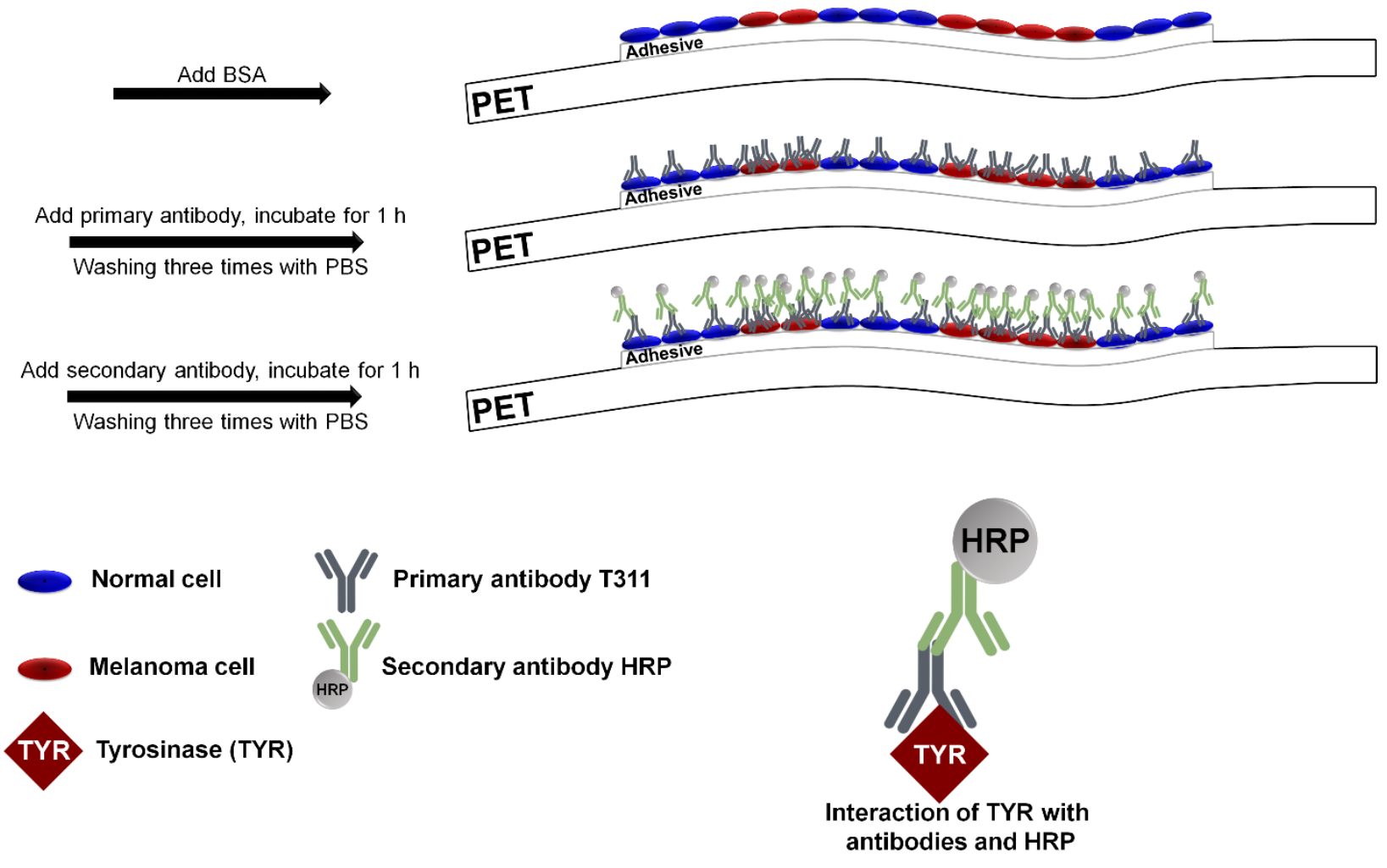

Figure S-2. Schematic of immunostaining of cells on and adhesive layer. 
SI-2. Microscopic images of melanoma cells

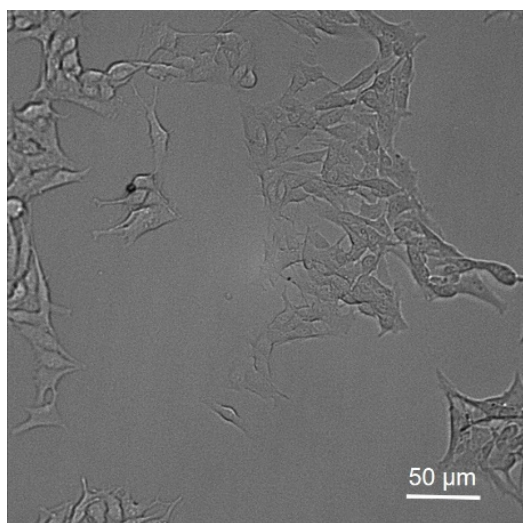

Figure S-3. Microscopic image of B16 melanoma cell culture in Petri dish.
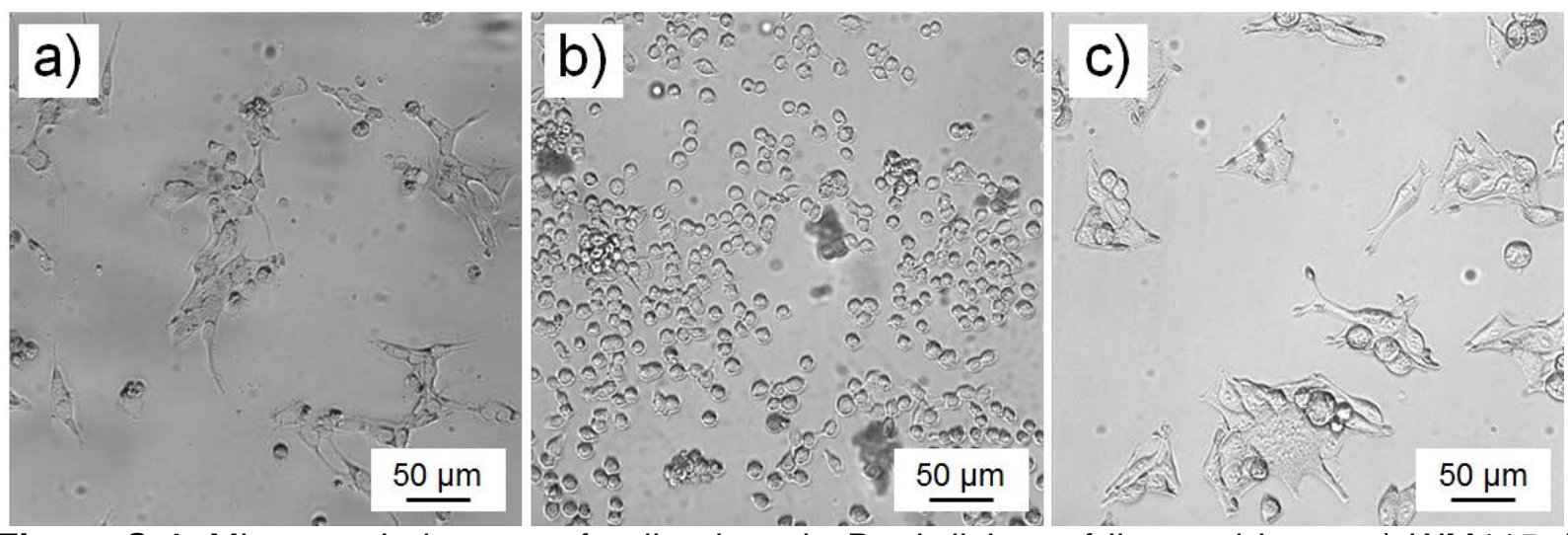

Figure S-4. Microscopic images of cell culture in Petri dishes of live and intact a) WM115, b) SBcl2 and c) WM239 cells. 
Tape-collected corneocytes
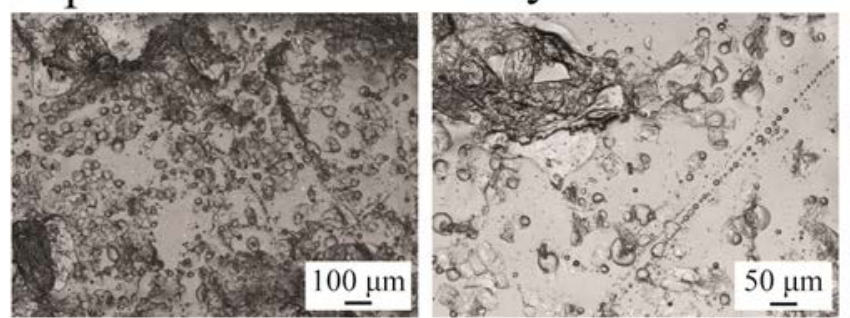

WM115
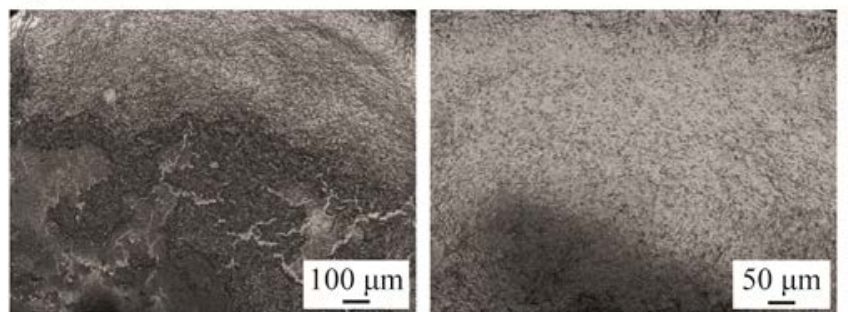

WM239
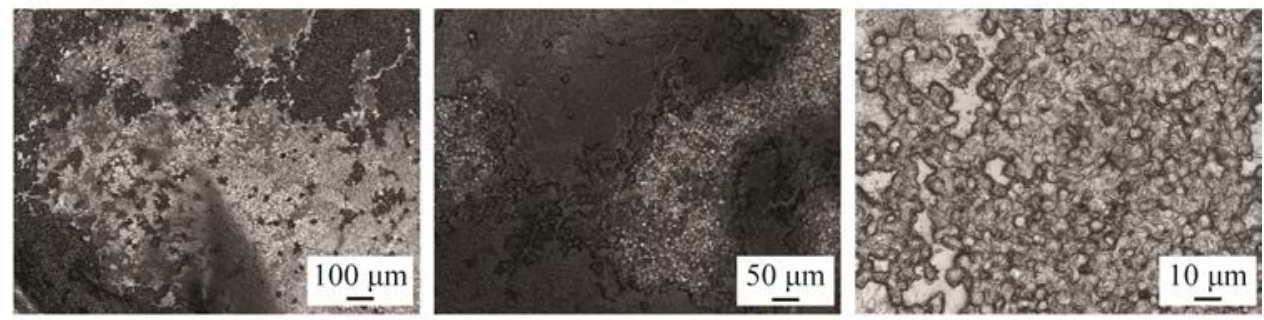

$\mathrm{SBcl} 2$
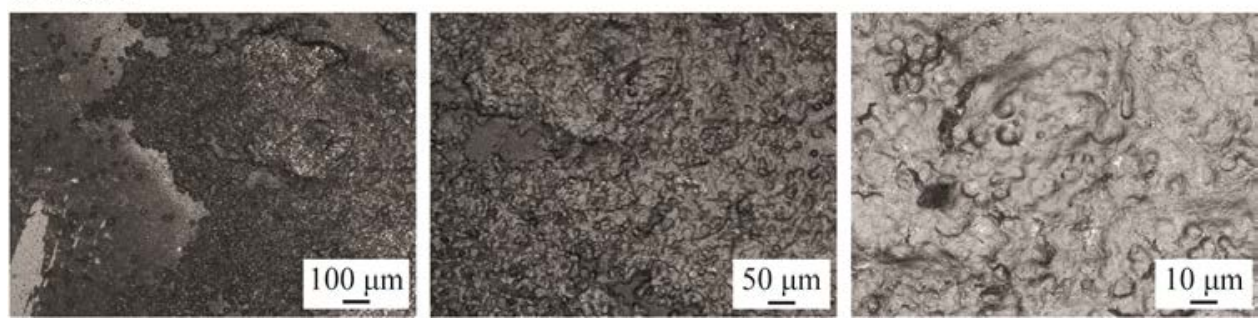

Figure S-5. Laser scanning micrographs of WM115, SBcl2, WM239, and cells collected from the stratum corneum after tape collection and pressing. 
SI-3. Soft microelectrode and schematic representation of SECM line scans on the taped samples.

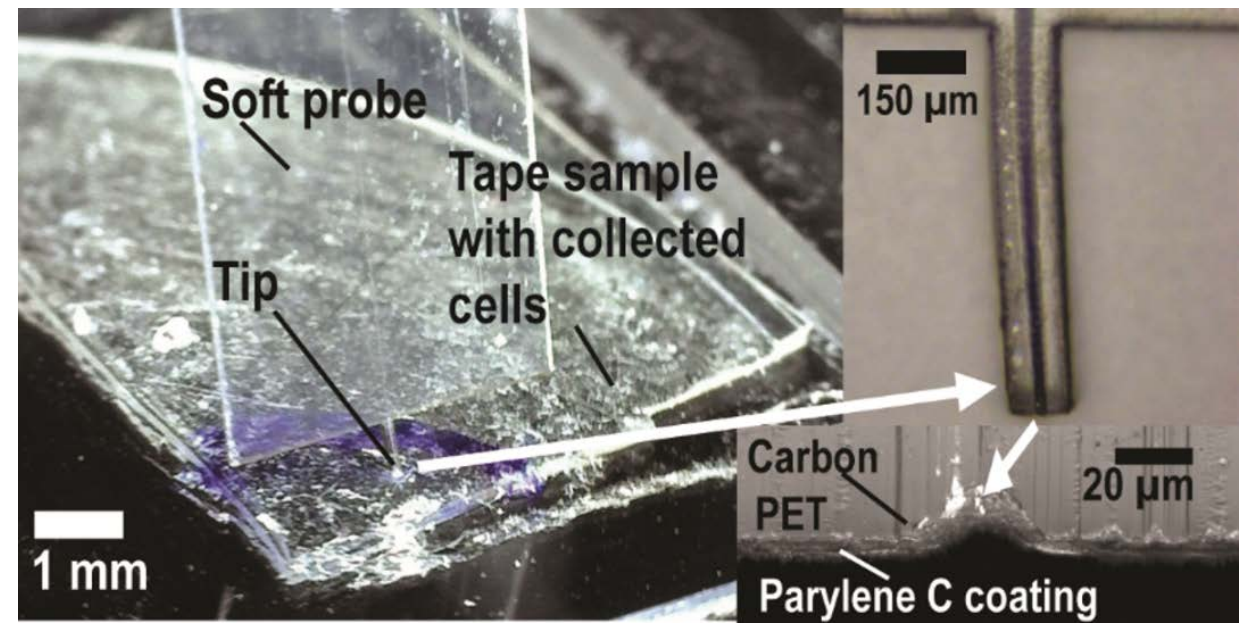

Figure S-6. Optical micrographs of a soft SECM probe on an adhesive tape with collected cells (insets show the side view and the cross-sectional view of the tip). Note: blue marks are guides to the eye on the backside of the tape.
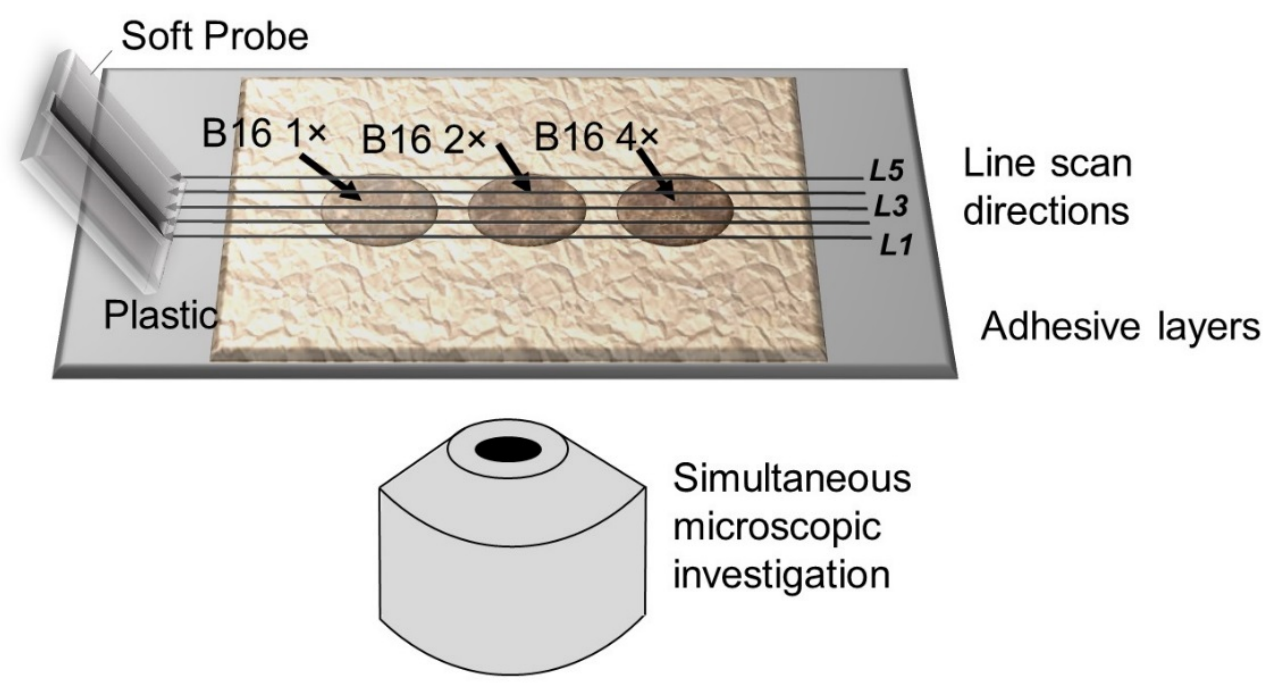

Figure S-7. Schematic of Soft-Probe-SECM line scan directions on a sample with normal mouse skin and B16 cells of different relative concentrations. Number of lines scans $L$ were either 1-5 or 1-10. The scanning direction was from right to left and line scan 3 (L3) and line scans 5-6 (L5, L6) were located near the center of all patterns for experiments with 5 and 10 line scans, respectively. 
SI-4. Cyclic voltammogram of a soft microelectrode in FcMeOH solution.

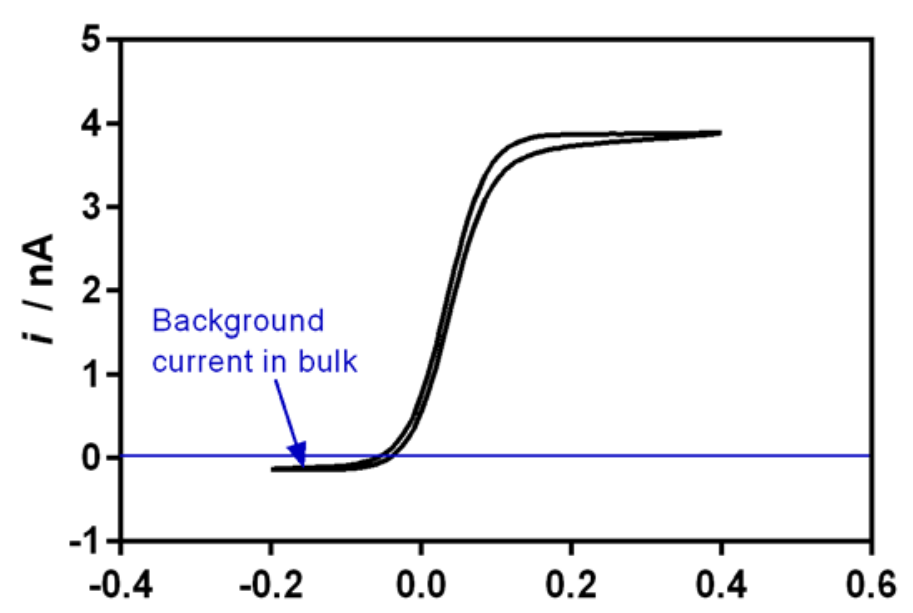

Figure S-8. Cyclic voltammetry of a soft microelectrode in $2 \mathrm{mM} \mathrm{FcMeOH}$ and $1 \mathrm{mM} \mathrm{H}_{2} \mathrm{O}_{2}$ in PB $(\mathrm{pH} 6)$. Scan rate $=50 \mathrm{mV} / \mathrm{s}$. 


\section{SI-5. Soft-Probe-SECM line scans over cells on adhesive tape with statistical analysis}

All data are presented as the mean \pm the standard deviation.

For statistical analysis, the SECM data from the analysis of the patterns of the normal mouse skin and the B16 melanoma cell line, the melanoma mice samples at different stages of tumor growth and simulated human skin sample (WM239, WM115, SBcl2 cells, normal skin and plastic) were analyzed with one-way ANOVA. The null hypothesis for all statistical analyses was that two sample means were identical.

Due to the variances that can appear between different SECM experiments, for instance due to slight variations in electrode area and soft probe angle as a result of the mechanical cutting of the SECM tip, the test results of the mouse melanoma samples were only reported versus the corresponding normal skin region of the same week. Indeed, comparing the mean values of the normal skin samples of weeks 3 and 4.5 and for weeks 4.5 and 6 showed significant differences.

For all analyzes, $\mathrm{p}<0.05$ was considered statistically different. 


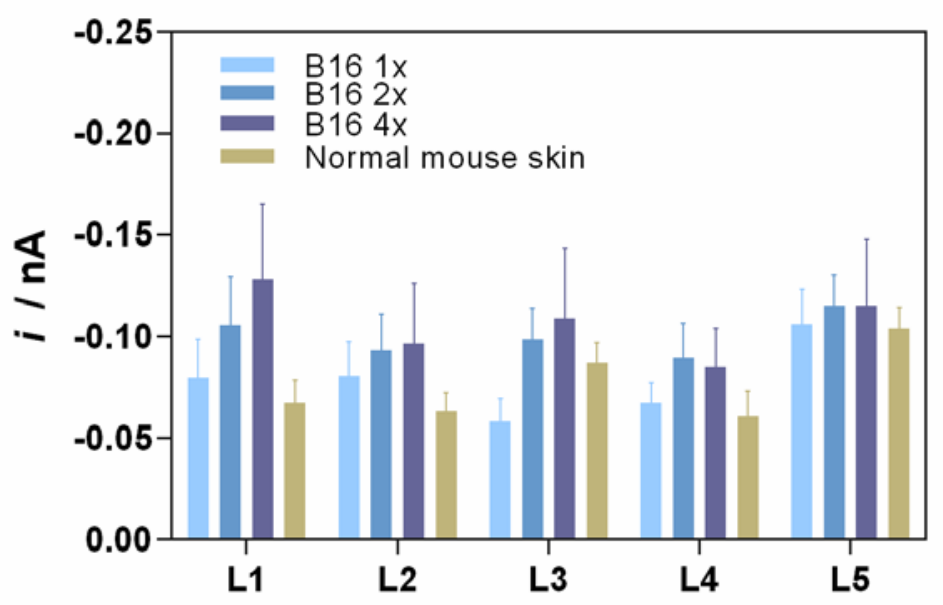

Figure S-9. Mean values \pm the standard deviation of each of the five line scans of Figure 2 - B16 melanoma mouse cell samples.

Table T-1. Details about SECM data of B16 melanoma mouse cell samples (Figure 2):

\begin{tabular}{cccc}
\hline Pattern & $\begin{array}{c}\text { Mean current } I \\
\mathbf{n A}\end{array}$ & $\begin{array}{c}\text { Std. Dev.I } \\
\mathbf{n A}\end{array}$ & No. of data points \\
\hline $\begin{array}{c}\text { Normal mouse } \\
\text { skin }\end{array}$ & -0.073 & 0.019 & 910 \\
B16 1x & -0.076 & & \\
B16 2x & -0.099 & 0.021 & 435 \\
B16 4x & -0.105 & 0.021 & 595 \\
\end{tabular}




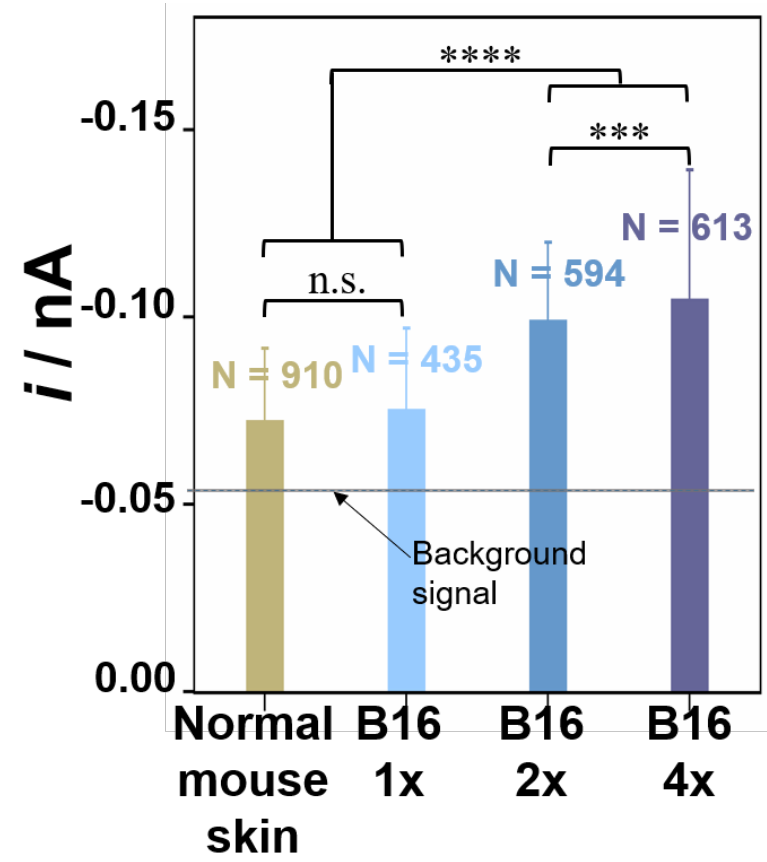

Figure S-10. Mean currents \pm the standard deviation from the five line scans of Figure 2 and background current on bare plastic. $\mathrm{N}$ indicates the number of data points for each sample region in this experiment. Meanings: n.s. not significant, ${ }^{\star \star \star} p<0.001$ and ${ }^{\star \star * \star} p<0.0001$.

Table T-2. Details about SECM data of the mice skin samples (Figure 4):

\begin{tabular}{c|ccc|ccc}
\hline \multirow{2}{*}{$\begin{array}{c}\text { Time I } \\
\text { weeks }\end{array}$} & $\begin{array}{c}\text { Mean } \\
\text { current I }\end{array}$ & $\begin{array}{c}\text { Std. Dev. } \\
\text { I }\end{array}$ & $\begin{array}{c}\text { No. of } \\
\text { data }\end{array}$ & $\begin{array}{c}\text { Mean } \\
\text { current I }\end{array}$ & $\begin{array}{c}\text { Std. Dev. } \\
\text { I }\end{array}$ & $\begin{array}{c}\text { No. of } \\
\text { data }\end{array}$ \\
\hline $\mathbf{3}$ & -0.078 & 0.012 & 635 & -0.089 & 0.034 & 1067 \\
points & nA & nA & points \\
\hline $\mathbf{4 . 5}$ & -0.094 & 0.051 & 954 & -0.124 & 0.055 & 1434 \\
$\mathbf{6}$ & -0.089 & 0.048 & 1105 & -0.193 & 0.082 & 1794 \\
\hline
\end{tabular}




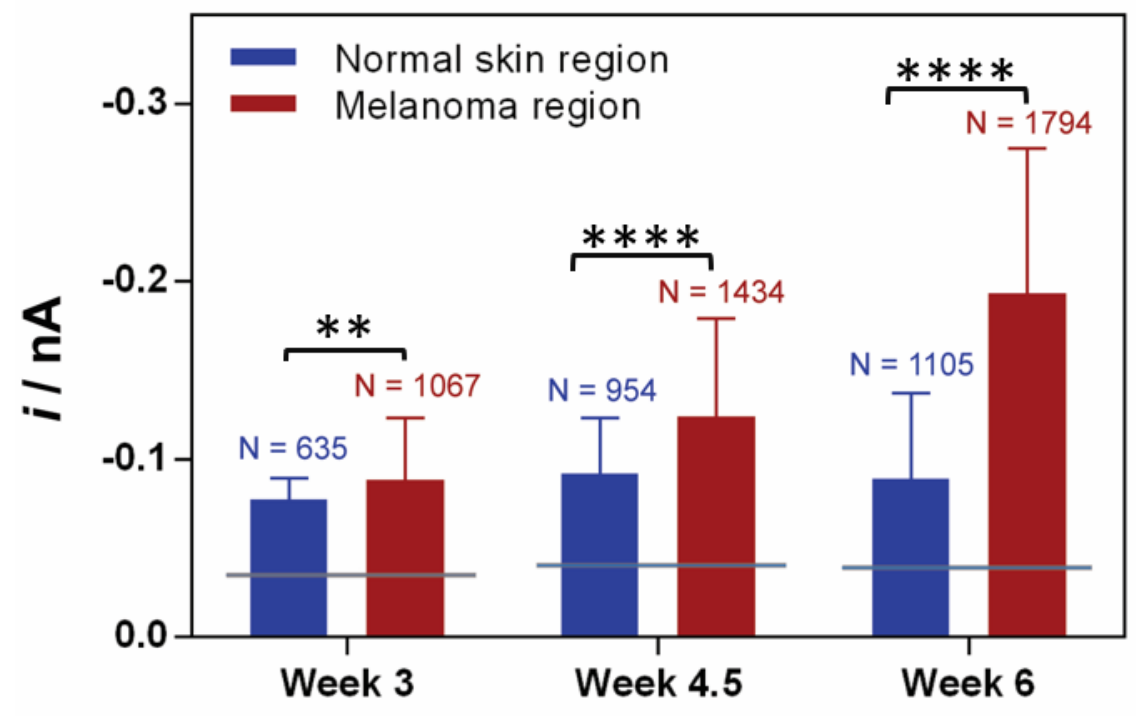

Figure S-11. Mean current \pm the standard deviation of electrochemical detection at different time periods after melanoma induction ( $3,4.5$ and 6 weeks) considering the data of Figure 4. $\mathrm{N}$ indicates the number of data points on the normal skin region and melanoma region of the same tapped skin. For each week, melanoma region and normal skin region of one mouse were investigated. Grey horizontal lines indicate the background SECM current in bare plastic. Meanings: ${ }^{* *} p<0.01,{ }^{* \star *} p<0.001$, and ${ }^{* \star * *} p<0.0001$.

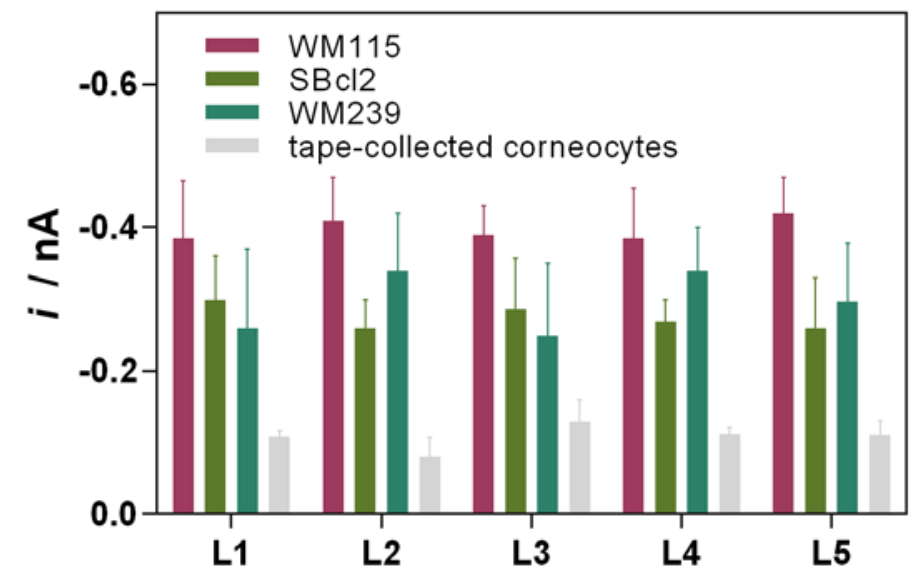

Figure S-12. Mean current values \pm the standard deviation of each of the five line scans of Figure 6 - human melanoma cell lines. 
Table T-3. Details about SECM data of human melanoma cell lines (Figure 6):

\begin{tabular}{cccc}
\hline Pattern & $\begin{array}{c}\text { Mean current } I \\
\text { nA }\end{array}$ & $\begin{array}{c}\text { Std. Dev.I } \\
\text { nA }\end{array}$ & No. of data points \\
\hline WM115 & -0.400 & 0.061 & 361 \\
SBc12 & -0.287 & 0.050 & 344 \\
WM239 & -0.292 & 0.082 & 365 \\
$\begin{array}{c}\text { Tape-collected } \\
\text { corneocytes }\end{array}$ & -0.108 & 0.019 & 728 \\
\hline
\end{tabular}

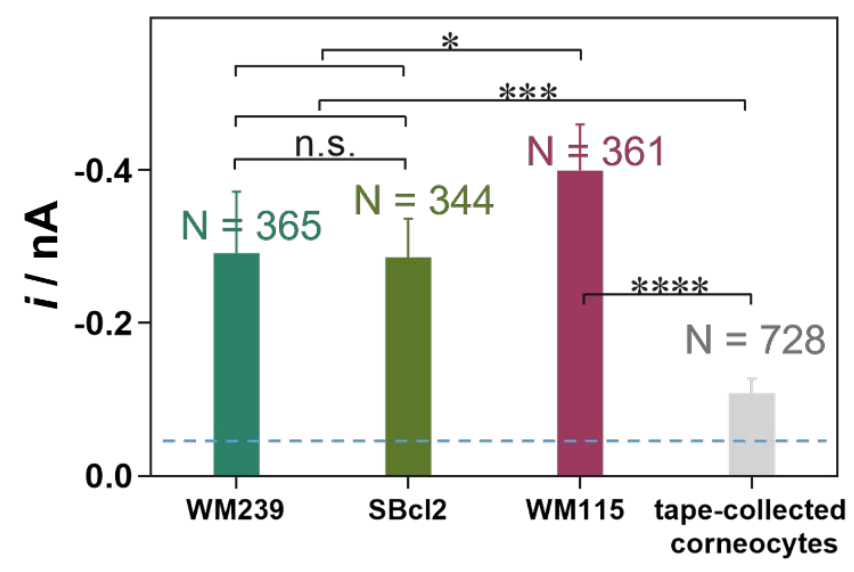

Figure S-13. Mean currents \pm the standard deviation from five line scans of Figure $6 . \mathrm{N}$ indicates the number of data points for each sample region in one experiment. Blue horizontal lines indicate the background SECM current on bare plastic. Meanings: n.s. not significant, ${ }^{*} p<0.05,{ }^{* \star *} p<$ 0.001 and ${ }^{* * *} p<0.0001$. 
SI-6. Colorimetric TYR visualization with TMB of human melanoma cell lines.

a)

b)

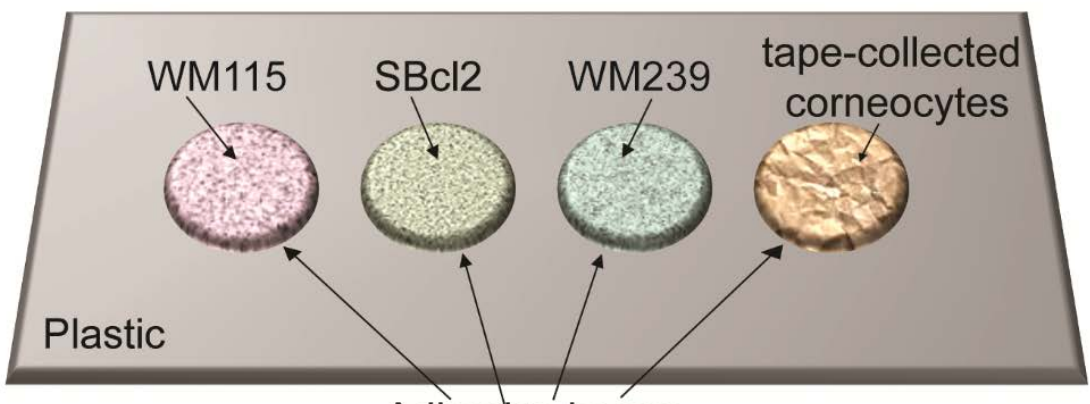

Adhesive layers

Before TYR assay with TMB
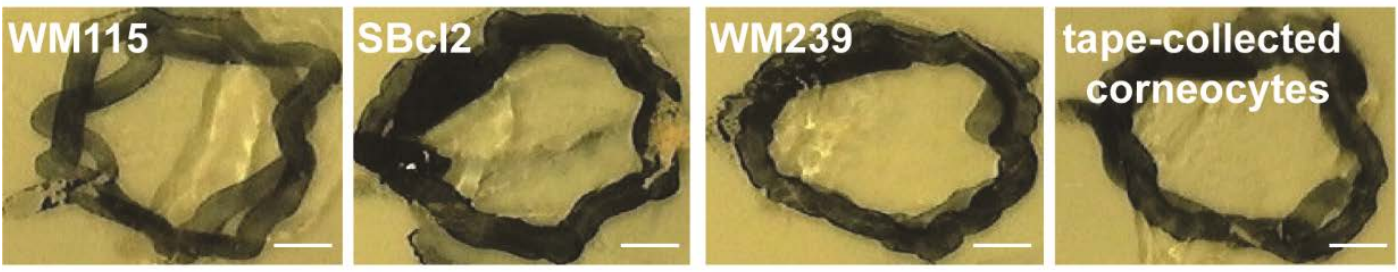

TYR assay with TMB (2 min)
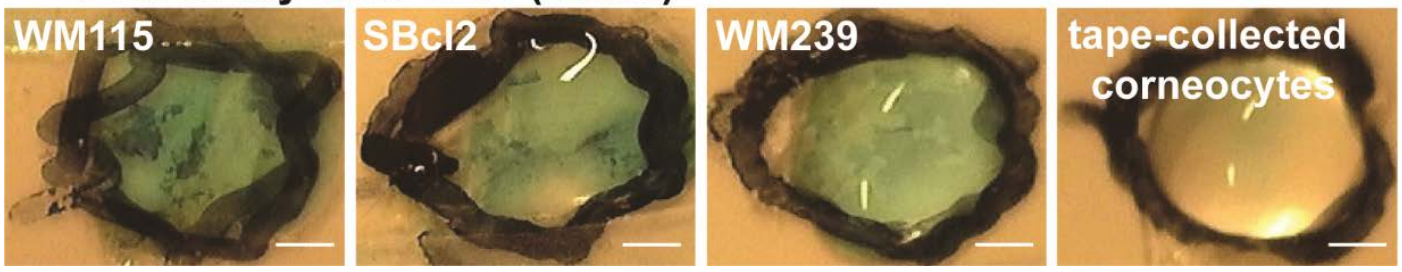

Figure S-14. Exemplary sample with human melanoma cells lines WM115, SBcl2 and WM239, and tape-collected corneocytes cells after tape collection and pressing. a) Schematic representation of sample design. b) Colorimetric visualization. Note: Black marks are on the back of the tape as guides to the eye. 


\section{SI-7. Colorimetric TYR visualization with TMB substrate for viable melanoma cell detection with intact cell membranes}

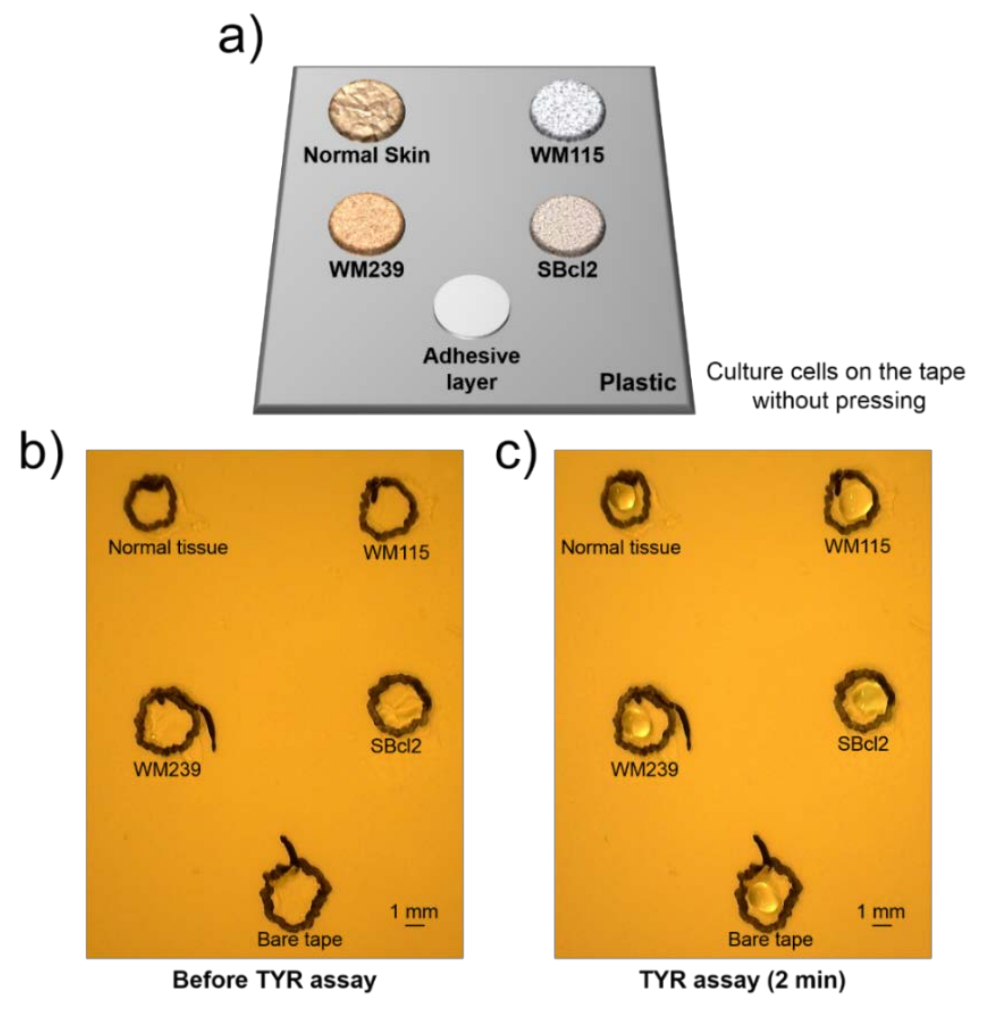

Figure S-15. Colorimetric visualization of three melanoma cell lines on adhesive tape without mechanical pressing of the cells. a) Schematic of the sample design. b) Before and d) after 2 min of the colorimetric TYR assay using TMB as reagent. Note: black lines in (c) and (d) were drawn on the back of the tape as guides to the eye in order to indicate the location of the cell patterns.

The TYR immunoassay was applied to intact cells for colorimetric detection. The schematic in Fig. S15a shows the design of the sample with WM115, WM239 and SBcl2 melanoma cells, dead normal skin cells and a bare adhesive layer on a PET foil. The macroscopic image of the adhesive tapes before and 2 min after covering the cell patterns with TMB solution are shown in Fig. S15b and c. The color of the solution above the cell patterns did not turn to blue confirming that the antibodies did not access the TYR inside the cells. In addition, the immunoassay test on the bare adhesive layer confirmed that non-specific binding of the antibodies to the adhesive layer was excluded (no color change). 